Article

\title{
Mg-Zn-Ca Alloys for Hemostasis Clips for Vessel Ligation: In Vitro and In Vivo Studies of Their Degradation and Response
}

\author{
Yen-Hao Chang ${ }^{1}$, Chun Chieh Tseng ${ }^{2}$, Chih-Yeh Chao ${ }^{3}$, Chung-Hwan Chen ${ }^{4,5,6,7,8}$, \\ Sung-Yen Lin $4,5,6,7,8$ and Je-Kang Du 1,9,*(D) \\ 1 School of Dentistry, College of Dental Medicine, Kaohsiung Medical University, Kaohsiung 80708, Taiwan; \\ edward590198@gmail.com \\ 2 Combination Medical Device Technology Division, Medical Devices and Opto-Electronics Equipment \\ Department, Metal Industries Research \& Development Centre, Lujhu Township, Kaohsiung 82151, Taiwan; \\ cctseng0915@gmail.com \\ 3 Department of Mechanical Engineering, National Pingtung University of Science and Technology, \\ Pingtung 91201, Taiwan; cychao@mail.npust.edu.tw \\ 4 Orthopedic Research Center, Kaohsiung Medical University, Kaohsiung 80708, Taiwan; \\ hwan@kmu.edu.tw (C.-H.C.); tony8501031@gmail.com (S.-Y.L.) \\ 5 Department of Orthopedics, College of Medicine, Kaohsiung Medical University, Kaohsiung 80708, Taiwan \\ 6 Division of Adult Reconstruction Surgery, Department of Orthopedics, \\ Kaohsiung Medical University Hospital, Kaohsiung Medical University, Kaohsiung 80708, Taiwan \\ 7 Department of Orthopedics, Kaohsiung Municipal Ta-Tung Hospital, Kaohsiung Medical University, \\ Kaohsiung 80145, Taiwan \\ 8 Regeneration Medicine and Cell Therapy Research Center, Kaohsiung Medical University, \\ Kaohsiung 80708, Taiwan \\ 9 Department of Dentistry, Kaohsiung Medical University Hospital, Kaohsiung 80708, Taiwan \\ * Correspondence: dujekang@gmail.com; Tel.: +886-7-3121101
}

Received: 14 June 2020; Accepted: 1 July 2020; Published: 7 July 2020

check for updates

\begin{abstract}
To control the degradation rate of magnesium (Mg) alloys, chitosan (CHI) and L-glutamic acid (LGA) were used as coatings on Mg-Zn-Ca alloys via dip coating. In this study, either two or seven CHI/LGA layers were applied as a coating on Mg-2.8Zn-0.8Ca alloy (ZX31) and Mg-2.8Zn-0.8Ca hemostasis clips (ZX31 clips). The morphologies, compositions, and surface roughness of the specimens were characterized via scanning electron microscopy, Fourier transform infrared spectroscopy, and surface measurement devices. The degradation rates and behavior of the specimens were evaluated by immersing them in simulated body fluids and by applying these ZX31 clips on rabbits' uterine tubes for five weeks. The specimen with seven layers $\left(\mathrm{ZX} 31(\mathrm{CHI} / \mathrm{LGA})_{7}\right)$ exhibited improved corrosion behavior when compared with ZX31 or ZX31(CHI/LGA) 2 , with a reduced degradation rate of the $\mathrm{Mg}$ alloy in a simulated body environment. In vivo experiments showed that ZX31 clips exhibited good biocompatibilities in each group but could not maintain the clamping function for five weeks. The weight loss of ZX31(CHI/LGA) 7 was significantly lower than that of the other groups. Consequently, it was verified that $\mathrm{CHI}$ can be used as a protective layer on a magnesium alloy surface via in vitro and in vivo experiments.
\end{abstract}

Keywords: Mg-Zn-Ca alloy; hemostasis clips; chitosan; L-glutamic acid; dip coating

\section{Introduction}

Laparoscopic surgery (LS) has become a common surgical procedure because of its advantage in reducing the probability of injury, while causing little or no wound complications; consequently, 
this results in short hospital stays after the surgery [1-3]. During abdominal LS operations, hemostasis clips are one of the most common devices used for vessel ligation, replacing sutures. In commercial hemostasis clips, the most commonly used materials are titanium (Ti) or titanium alloys (Ti alloys) because of their biocompatibility, high strength, and good corrosion resistance [1,2]. However, their use may cause artefacts during magnetic resonance imaging (MRI) and in computed tomography (CT) scans because of the high X-ray absorption coefficient of $\mathrm{Ti}$ [1-3]. Case reports have also shown that clip migration (CM) can occur in some patients after surgery [4-6]. In addition to patient suffering and the requirement of additional surgery, CM is likely to cause other complications [6]. To overcome these problems, bio-absorbable polymer clips have recently been developed. However, the application of polymer clips has been limited due to their low strength [1].

Magnesium (Mg) and magnesium alloys (Mg alloys) have become the most prominent metallic biomaterials in recent years due to their biodegradability and good biocompatibility. Mg alloys can be absorbed by the body and eliminated via urine due to their chemical reaction with $\mathrm{H}_{2} \mathrm{O}$. Furthermore, $\mathrm{Mg}$ and $\mathrm{Mg}$ alloys have many attractive physical characteristics, such as their density $\left(1.74-2.0 \mathrm{~g} / \mathrm{cm}^{3}\right)$ and Young's modulus (approximately 41-45 GPa) [7]. However, $\mathrm{Mg}$ alloys corrode too quickly when they are exposed to body fluids. The human body cannot absorb the entire amount of produced hydrogen, thus gas pockets are generated and bubbles are accumulated [8]. Additionally, uneven corrosion could result in corrosion-assisted cracking and loss of strength (risk of implant failure), as well as locally high $\mathrm{pH}$ values [9]. Among these, locally high $\mathrm{pH}$ values cause surrounding cells to undergo osmotic shock, resulting in tissue inflammation [9]. This limits the clinical applications of $\mathrm{Mg}$ alloys as medical implant devices.

Numerous approaches have been proposed in the literature to improve corrosion resistance, such as alloying, cryogenic machining, and equal channel angular pressing (ECAP) [10,11]. In addition, surface modification of $\mathrm{Mg}$ alloys is also an effective approach for improving the corrosion resistance, such as micro-arc oxidation (MAO) coating, chemical conversion coating, electrophoretic deposition coating, and atomic layer deposition [12-17]. Furthermore, various polymers have been commonly used to coat the surfaces of $\mathrm{Mg}$ alloys to provide a protective layer and lower the corrosion rate (such as by dip coating). Among all the surface modification methods previously studied, the layer-by-layer (LbL) self-assembly technique is a simple and versatile method for incorporating polyelectrolytes, which is achieved by leveraging the electrostatic attractive forces between oppositely charged particles $[12,18]$.

Recently, hydrogels made of natural polymers, such as alginate, collagen, chitosan (CHI), gelatin, and L-glutamic acid (LGA), have been used in numerous fields, especially as biomaterials [19-21]. Among these polymers, CHI and LGA have many reported applications in different fields, such as tissue engineering, regenerative medicine, and drug delivery [22-28]. Thus, CHI can be regarded as a favorable material for coating onto magnesium alloys due to its biocompatibility, low toxicity, immune-stimulatory activity, and biodegradability [24,29]. In addition, CHI possesses adhesive properties and superior film-forming ability [30].

Liangjian et al. [31] used CHI as a coating on Mg-based composites. The results demonstrated that $\mathrm{CHI}$ coating creates an effective corrosion-resistant layer that can decrease $\mathrm{H}_{2}$ release. Additionally, the results indicated that the CHI coating did not exhibit cytotoxicity towards L-929 cells. However, the protective power of $\mathrm{CHI}$ on the surface is still slightly insufficient, and the corrosion resistance of magnesium alloys needs to be strengthened. In this context, it may be possible to control the degradation rate of $\mathrm{Mg}$ alloys by employing multiple $\mathrm{CHI}$ protective layers.

$\mathrm{CHI}$ becomes positively charged when $\mathrm{CHI}$ is dissolved in a solution with a pH value lower than its pKa. In order to create attractive forces between oppositely charged particles, LGA can be added, as it becomes negatively charged when the $\mathrm{pH}$ of the solution is higher than its $\mathrm{pKa}$. Furthermore, the amino group of LGA loses an extra proton, meaning LGA becomes doubly negative (-OOC-CH( $\left.\left(\mathrm{NH}_{2}\right)-\left(\mathrm{CH}_{2}\right)_{2}-\mathrm{COO}-\right)$ at $\mathrm{pH}$ values higher than 9.47. Thus, one of the negative charges of the LGA can adsorb the positively charged CHI and the other negative charge can adsorb the 
next positively charged CHI. In line with the above facts, polyelectrolyte multilayers (PEMs) can be fabricated by leveraging the electrostatic forces between CHI and LGA.

The aim of the present study is to fabricate a biocompatible Mg clip and assess the feasibility of realizing a multilayer coating using $\mathrm{CHI}$ as a polycation and LGA as a polyanion. The degradation characteristics of the fabricated clips were observed via in vitro and in vivo experiments.

\section{Materials and Methods}

\subsection{Materials and Specimen Preparation}

Mg-2.8Zn-0.8Ca alloy (ZX31) was produced from highly pure Mg (99.9\%), Zn (99.9\%), and Ca (99.8\%) using an electric resistance heating furnace with a mild steel crucible under argon atmosphere. ZX31 hot-extruded alloy bars (diameter, $10 \mathrm{~mm}$ ) were obtained at $350{ }^{\circ} \mathrm{C}$, with a reduction ratio of 10:8 via continuous extrusion (speed, $15 \mathrm{~cm} / \mathrm{min}$ ) of the ingot (diameter, $90 \mathrm{~mm}$ ). The as-extruded ZX31 alloy was cut into 3-mm-thick disks, which were used as specimens for the experiments. The chemical composition of the alloy is shown in Table 1. A rectangular line of $1 \mathrm{~mm} \times 0.8 \mathrm{~mm}$ was produced using the as-extruded ZX31 by further extrusion with the same parameters. Finally, the hemostasis clips were made using a self-made stamping machine. These clips were manufactured by the Metal Industries Research and Development Center (MIRDC, Kaohsiung, Taiwan) and are shown in Figure 1.

Table 1. Composition of the Mg-Zn-Ca alloy (wt.\%).

\begin{tabular}{|c|c|c|c|c|}
\hline Element & $\mathrm{Mg}$ & $\mathrm{Zn}$ & $\mathrm{Ca}$ & Al \\
\hline wt.\% & Bal. & 2.83 & 0.78 & 0.04 \\
\hline
\end{tabular}

Figure 1. Hemostatic Mg clips.

\subsection{Preparation of the Coating}

The specimens were ground with $\mathrm{SiC}$ paper of up to \#2000 and ultrasonically cleaned with ethanol. Before coating, the specimens were soaked in $5 \mathrm{M}$ of $\mathrm{NaOH}$ solution for $10 \mathrm{~min}$ with stirring to obtain hydroxylate $\left(\mathrm{MgOH}_{2}\right)$ on the surface. The specimens were again washed with ethanol and then dried with warm air. The surface of the alloy became negatively charged after etching [14]. CHI (medium molecular weight) and LGA were purchased from Sigma-Aldrich (St. Louis, MO, USA). A cationic $\mathrm{CHI}$ solution ( $0.6 \mathrm{wt} . \%$ ) was mixed with a $0.5 \%$ acetic solution to obtain the coating mixture. The $\mathrm{pH}$ was then adjusted to $5.0 \pm 0.2$ using $5 \mathrm{M}$ of $\mathrm{NaOH}$ solution. The $\mathrm{pH}$ of the anionic LGA solution $(10 \mathrm{~g} / \mathrm{L})$ was adjusted to 9.7 using $5 \mathrm{M}$ of $\mathrm{NaOH}$ solution. First, the pretreated specimens were dip-coated in the $\mathrm{CHI}$ solution for $10 \mathrm{~min}$ and dried at $110^{\circ} \mathrm{C}$ for $1 \mathrm{~h}$. Thereafter, the specimens were dip-coated in the LGA solution for $1 \mathrm{~min}$ and dried at $110^{\circ} \mathrm{C}$ for $1 \mathrm{~h}$. A schematic illustration of the coating process is shown in Figure 2. The number of layers of $\mathrm{CHI}$ coating was used to name the samples. In this study, samples with either two or seven layers of $\mathrm{CHI}$ were produced via the aforementioned dip coating process. The samples were denoted as substrate ZX31 (CHI/LGA) $)_{2}$ or ZX31 (CHI/LGA) 7 . 


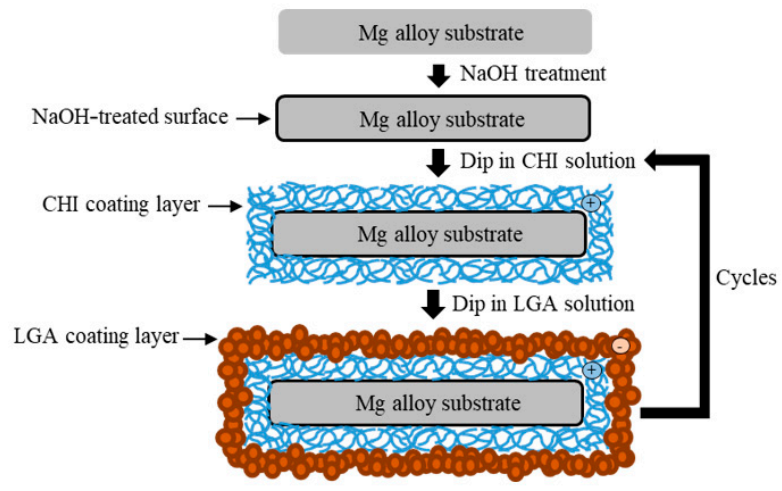

Figure 2. Schematic illustration of the chitosan (CHI) and L-glutamic acid (LGA) coating process.

\subsection{Surface Characterization}

First, the coated surface of the specimen was gold-sputtered. Then, a scanning electron microscope (SEM) (S-3000N, Hitachi, Tokyo, Japan) with an energy-dispersive spectrometer (EDS) (E-MAX ENERGY, HORIBA, Kyoto, Japan) was used to examine the surface morphology and chemical composition of the samples at $15 \mathrm{kV}$. The surface characteristics and roughness of the coating layers were investigated using a 3-D surface measurement device (GBS, SmartWLI-Prime, Ilmenau, Germany). The roughness was measured using the surface roughness parameter $\mathrm{Ra}$, which is the arithmetic average of the surface height values. Fourier transform infrared spectroscopy (FTIR) (MER Spectrum 100 FTIR, PerkinElmer, Waltham, MA, USA) was performed to analyze the chemistry of the CHI and LGA that were coated onto the ZX31 samples. Pellets of CHI and LGA were formed by compressing the samples in a stainless steel mold at high pressure. The spectra were calculated from 600 to $4000 \mathrm{~cm}^{-1}$, with a resolution of $4 \mathrm{~cm}^{-1}$.

\subsection{In Vitro Degradation Tests}

The prepared samples were immersed in simulated body fluid (SBF) [32] at $37^{\circ} \mathrm{C}$ for 30 days. In order to maintain a relatively stable immersion environment in vitro, the SBF solution was renewed every $24 \mathrm{~h}$. Three samples were tested for each set of conditions. The hydrogen evolution method [28] was used to calculate the volume of hydrogen released. Using this method, hydrogen gas was collected and the amount of hydrogen released was calculated via the measured height difference of the liquid levels. The weight loss of the samples was used to calculate the corrosion rate ( $\mathrm{mm} / \mathrm{year})$ through the following equation [33]:

$$
\text { Corrosion rate }=(\mathrm{K} \times \mathrm{W}) /(\mathrm{A} \times \mathrm{T} \times \mathrm{D})
$$

where $\mathrm{K}$ is a coefficient with a value of $8.76 \times 10^{4}, \mathrm{~W}$ is the weight loss $(\mathrm{g}), \mathrm{A}$ is the area of the sample exposed to solution $\left(\mathrm{cm}^{2}\right), T$ is the exposure time $(\mathrm{h})$, and $\mathrm{D}$ is the density of the material $\left(\mathrm{g} / \mathrm{cm}^{3}\right)$.

\subsection{Animal Surgery Study}

Animal experiments were conducted in compliance with animal ethics regulations and were authorized by the Institutional Animal Care and Use Committee (No. 106170). Three adult female New Zealand rabbits weighing 3.0-5.0 kg each were used in the study to simulate the implantation of $\mathrm{Mg}$ clips during human surgery. We selected the rabbit's uterine tube to simulate the implantation of the hemostatic clips because of its appropriate size. The animals were administered general anesthesia before the surgery, and the region to be operated on was scrubbed with a $25 \mathrm{~g} / \mathrm{L}$ tincture of iodine and $70 \%$ ethanol. Afterwards, we randomly selected a rabbit and named it rabbit- 1 . Continuous hemostatic clips were applied on the left and right uterine tubes of rabbit-1. Three uncoated ZX31 clips and three ZX31(CHI/LGA) 2 clips were attached to the left and right uterine tubes, respectively, of this rabbit. The uterine tubes were surgically put back into the animal and the wound was 
sutured. Then, we randomly selected a second rabbit (rabbit-2) and applied three ZX31 and three $\mathrm{ZX} 31(\mathrm{CHI} / \mathrm{LGA})_{7}$ clips to its left and right uterine tubes, respectively.

Finally, the left and right uterine tubes of the third rabbit were clipped with three ZX31(CHI/LGA) and three $\mathrm{ZX} 31(\mathrm{CHI} / \mathrm{LGA})_{2} \mathrm{Mg}$ clips, respectively. The three rabbits were euthanized five weeks after surgery.

\subsection{Histological and Radiographic Evaluations}

Radiographs were obtained to observe the degradation process at two and three weeks after surgery. After the removal of the Mg clips, the soft tissue of the oviduct samples was dehydrated. The tissues were then embedded in paraffin and a histological evaluation was performed on hematoxylin-and-eosin-stained sections. The weight loss of each of the $\mathrm{Mg}$ clips was also measured using a precision balance (AS 220/C/1, RODWAG, Radom, Poland); this measurement was performed before and after the surgery.

\subsection{Statistical Analysis}

One-way analysis of variance (ANOVA) testing was used to determine the level of significance, and multiple comparisons were performed using Scheffe's method. Statistical differences were considered to be significant at $p<0.05$.

\section{Results and Discussion}

\subsection{Surface Morphology}

Figure 3 shows the SEM morphologies and 2-D texture height maps of the ZX31, ZX31(CHI/LGA) and ZX31(CHI/LGA $)_{7}$ specimens. The uncoated specimen had visible scratches, which were attributed to the grinding process (Figure 3a), and the surface roughness ( $\mathrm{Ra}$ ) was $1.19 \mu \mathrm{m}$. The specimens coated with $\mathrm{CHI}$ are shown in Figure 3b,c. In contrast, there were no scratches on the $\mathrm{CHI}$-coated $\mathrm{Mg}$ specimens (Figure 3b), indicating that the films fully covered the substrates. The ZX31(CHI/LGA) and $\mathrm{ZX31}(\mathrm{CHI} / \mathrm{LGA})_{7}$ specimens exhibited some $\mathrm{CHI}$ agglomerations on the substrate, and the agglomerations of the ZX31(CHI/LGA $)_{7}$ specimen were larger than those of the ZX31(CHI/LGA) 2 specimen. Mainly because of the coating process, the surfaces of initially small agglomerations continued to have $\mathrm{CHI}$ deposited onto them. As the number of electrodeposition layers increased, the height of the surface increased. The surface roughness of the $\mathrm{CHI}$-coated specimens increased from $1.72 \mu \mathrm{m}$ (two layers) to $2.60 \mu \mathrm{m}$ (seven layers). The surface roughness of the CHI-coated specimens was high due to the different deacetylation degrees of $\mathrm{CHI}$ molecules and the corrosion of the $\mathrm{Mg}$ substrate during film formation [30].

\subsection{Coating Composition}

FTIR was employed to analyze the chemical composition of the surface of the coating (Figure 4). For comparison purposes, the FTIR spectrum of pure CHI was also measured. The spectra of the $\mathrm{ZX} 31(\mathrm{CHI} / \mathrm{LGA})_{2}$ and ZX31(CHI/LGA $)_{7}$ specimens exhibited similar characteristic peaks to those of their parent polymers. In the spectrum of the $\mathrm{ZX} 31(\mathrm{CHI} / \mathrm{LGA})_{7}$ specimen, the characteristic saccharide peaks of $\mathrm{CHI}$ in the $961-1186 \mathrm{~cm}^{-1}$ region were observed. The $\mathrm{C}-\mathrm{O}$ stretching absorption band at $1065 \mathrm{~cm}^{-1}$ and $1027 \mathrm{~cm}^{-1}$ and the C-O-C asymmetric stretching vibrations at $1149 \mathrm{~cm}^{-1}$ were also observed. The broad absorption line in the region between 3200 and $3600 \mathrm{~cm}^{-1}$ was attributed to -OH and -NH stretching, and the signals at approximately 1645,1555 , and $1378 \mathrm{~cm}^{-1}$ were attributed to the amide I, II, and III modes of the residual $\mathrm{N}$-acetyl groups, respectively. In the coated specimens, high-frequency shifts of amide II were found, which were ascribed to the electrostatic interaction between the $-\mathrm{COOH}$ of LGA and the $-\mathrm{NH}_{2}$ of $\mathrm{CHI}$ [34]. This phenomenon was present with a more dramatic shift in the $\mathrm{ZX} 31(\mathrm{CHI} / \mathrm{LGA})_{7}$ specimen. Therefore, the results of the FTIR analysis 
clearly indicated the successful fabrication of CHI and LGA multilayers. Moreover, the spectra of the specimens also varied with the number of layers of $\mathrm{CHI}$ coating.
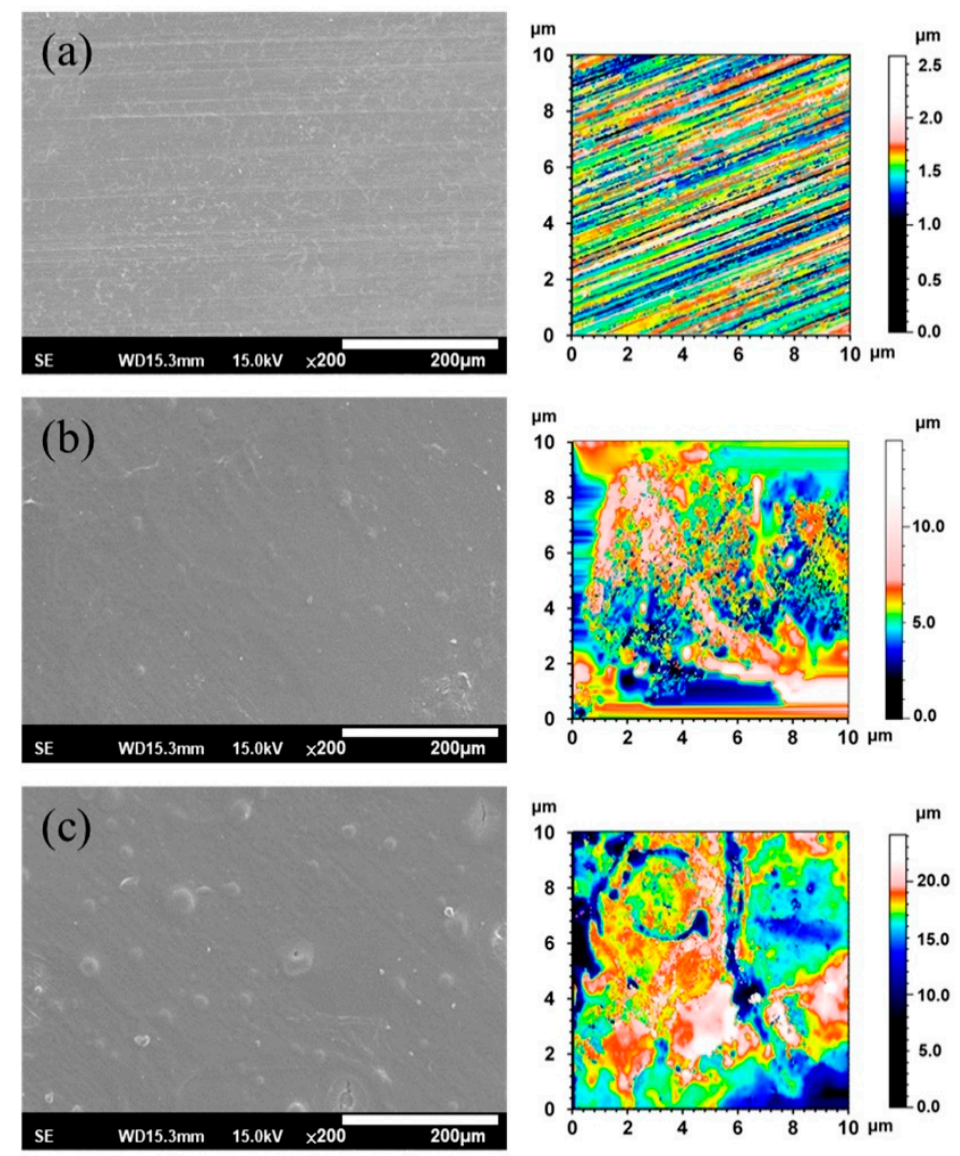

Figure 3. SEM and 2-D texture height maps of (a) a specimen ground with \#2000 $\mathrm{SiC}$ paper; (b) ZX31(CHI/LGA) 2 specimen; (c) ZX31(CHI/LGA) 7 specimen.

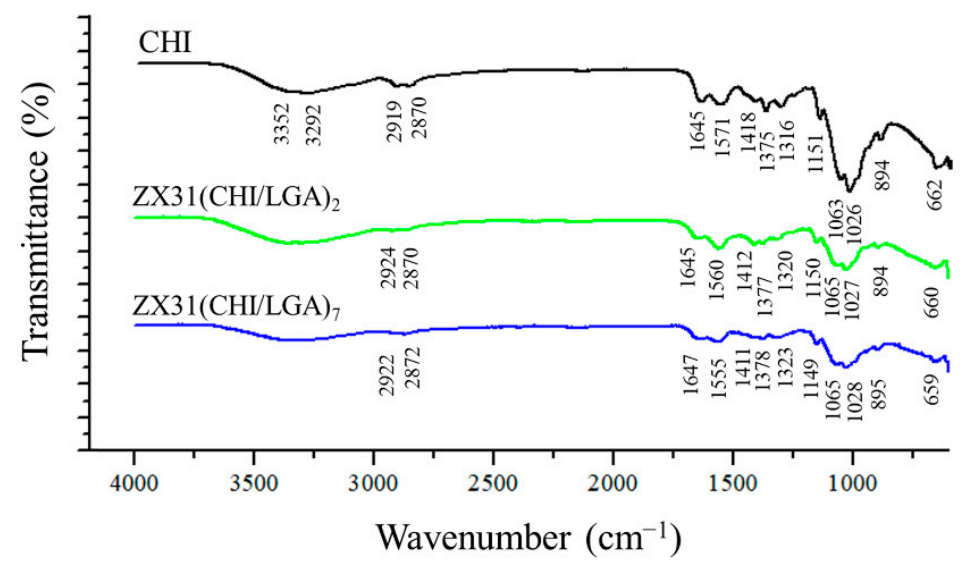

Figure 4. FTIR spectra of pure $\mathrm{CHI}, \mathrm{ZX} 31(\mathrm{CHI} / \mathrm{LGA})_{2}$ and $\mathrm{ZX} 31(\mathrm{CHI} / \mathrm{LGA})_{7}$ specimens.

\subsection{In Vitro Corrosion Resistance}

The degradation rates of ZX31 specimens with or without a chitosan coating were measured in simulated body fluid (SBF) at $37 \pm 0.2{ }^{\circ} \mathrm{C}$ for 30 days. The released hydrogen $\left(\mathrm{H}_{2}\right)$ volumes of the different specimens were measured to analyze the effect of the degradation rate of the coating and understand its corrosion behavior. The degradation rates of the different specimens are shown in Figure 5a. It can be seen that uncoated ZX31 exhibited a high degradation rate during the 30 days of 
immersion. Moreover, it can be seen that the degradation rates of the $\mathrm{CHI}$-coated specimens were markedly low during this period. The average degradation rates of the ZX31, ZX31(CHI/LGA) and ZX31(CHI/LGA) 7 specimens were $5.12,4.11$, and $3.03 \mathrm{~mm} / \mathrm{y}$, respectively. The specimen with seven layers of $\mathrm{CHI}$ coating exhibited a lower degradation rate when compared with that for the specimen with two layers. The improved corrosion resistance of the ZX31(CHI/LGA $)_{2}$ and ZX31(CHI/LGA) 7 specimens when compared with the ZX31 specimen can be attributed to the use of polyelectrolyte multilayers (PEMs). Moreover, an increase in the number of layers of $\mathrm{CHI}$ coating can delay the corrosion of the $\mathrm{Mg}$ substrate in corrosive environments.
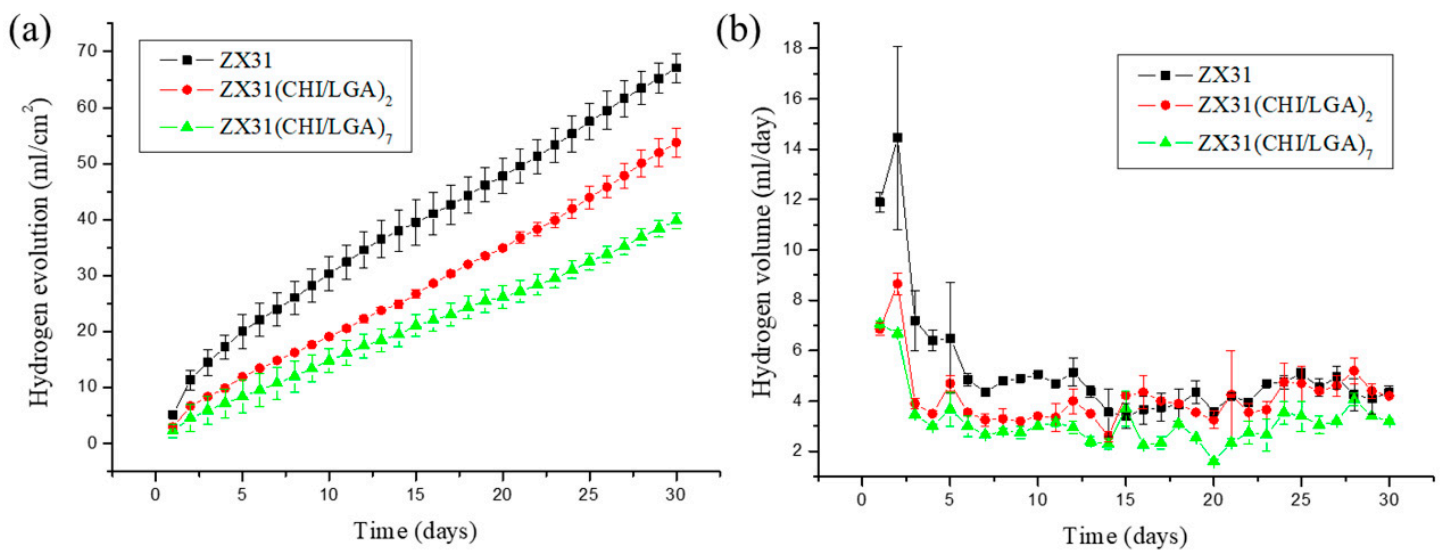

Figure 5. Volume of hydrogen for each of the specimens during a 30-day immersion test in SBF: (a) hydrogen evolution process of uncoated ZX31, ZX31(CHI/LGA) $)_{2}$, and ZX31(CHI/LGA) 7 specimens immersed in an SBF solution at $37 \pm 0.2^{\circ} \mathrm{C}$ for 30 days; (b) $\mathrm{H}_{2}$ released per day.

Figure $5 \mathrm{~b}$ shows that the uncoated $\mathrm{ZX} 31$ released a higher amount of $\mathrm{H}_{2}$ per day than the coated specimens during the first immersion stage of seven days. It can be noted that the amount of $\mathrm{H}_{2}$ released per day was similar in the ZX31 and ZX31(CHI/LGA) 2 specimens, as shown in Figure 5b. After seven days of immersion, the volume of $\mathrm{H}_{2}$ released was low and stable in all the specimens. This implies that the anticorrosion effect of $\mathrm{CHI}$ plays a significant role only in the early stage. The surface of the ZX31(CHI/LGA $)_{7}$ was completely covered by the $\mathrm{CHI}$, which acts as a protective layer to prevent environmental corrosion. After $\mathrm{CHI}$ degrades, the coated specimens lose their protection and their area of exposure increases, which consequently increases their degradation rate.

These in vitro results show that the degradation rate can be reduced by increasing the number of coating layers. Liangjian et al. [31] analyzed Mg-based composites that were coated with $\mathrm{CHI}$ and studied the in vitro degradation behavior in SBF. The results of their study showed that the CHI-coated specimens exhibited higher corrosion resistance than did the uncoated specimens. Moreover, the results of the in vitro cytotoxicity tests indicated that the $\mathrm{CHI}$-coated specimens were safe for medical use. Bai et al. [35] fabricated Mg-Zn-Ca alloys via MAO to produce porous surfaces. These specimens were dip-coated in a CHI solution to fill in the holes of the MAO surface. They concluded that CHI coating is a promising strategy for slowing down the degradation of Mg alloys. Furthermore, Cui et al. [12] produced an AZ31 (composition in mass fraction, \%: Al 2.5-3.5, Zn 0.6-1.4, Mn 0.2 and Balanced $\mathrm{Mg}$ ) alloy with $\mathrm{CHI}$ and poly-LGA coatings via LbL methods. The coated specimens that they used exhibited high corrosion resistance, as well as antibacterial properties. All these studies demonstrate the various methods employed for fabricating $\mathrm{CHI}$ coatings, which were a key factor for controlling the corrosion resistance of $\mathrm{Mg}$ alloys. This can be attributed to the fact that $\mathrm{CHI}$ undergoes relatively slow biodegradation in SBF (or physiological environments).

\subsection{EDS Analysis}

After the in vitro immersion test, the morphologies and elemental compositions of the degraded surfaces of the uncoated and CHI-coated specimens were studied using SEM and EDS. These 
results are shown in Figure 6. After 30 days of immersion, both the ZX31 and the ZX31(CHI/LGA) 2 specimens exhibited different surface morphologies with corrosion products on the surface. The rugged surface morphology of the ZX31 and ZX31(CHI/LGA) $)_{2}$ specimens revealed that severe degradation had occurred.

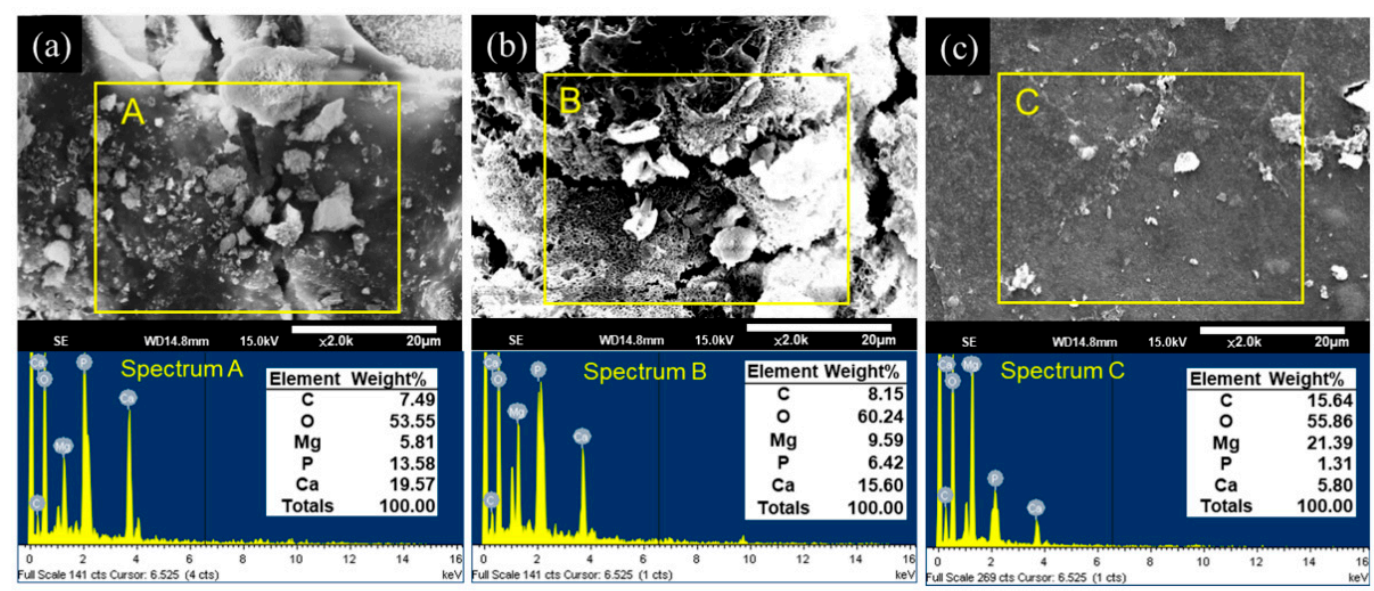

Figure 6. SEM observations and EDS point spectrum analysis of the corroded surfaces after 30 days of immersion for (a) ZX31; (b) ZX31(CHI/LGA) ${ }_{2}$ and (c) ZX31(CHI/LGA) 7 .

In addition, particles with various shapes and different sizes could be also seen on the surface of the specimens. EDS analysis showed that these particles were mainly composed of $\mathrm{Mg}, \mathrm{O}, \mathrm{C}, \mathrm{Ca}$, and P. Pan et al. [36] proved that these particles were bone-like apatites. Furthermore, the amount of corrosion products on the surface of the ZX31(CHI/LGA $)_{7}$ specimen after 30 days was lower than on the ZX31 and ZX31(CHI/LGA) 2 specimens. This implies that the ZX31(CHI/LGA) 7 specimen was more stable than the other two specimens.

\subsection{In Vivo Estimation}

Figure 7 shows the surgical procedures in which the Mg clips were used in the uterine tubes of each rabbit. Herein, Figure 7a,b displays the ZX31 and ZX31(CHI/LGA) 2 Mg clips on the left and right uterine tubes of rabbit- 1 after occlusion, respectively. Figure 7c,d displays the ZX31 and ZX31(CHI/LGA) 7 Mg clips on the left and right uterine tubes of rabbit-2 after occlusion, respectively. Moreover, Figure 7e,f displays the ZX31(CHI/LGA $)_{7}$ and ZX31(CHI/LGA) ${ }_{2} \mathrm{Mg}$ clips on the left and right uterine tubes of rabbit-3 after occlusion, respectively. The results show that the clips can completely close the uterine tubes of rabbits. All three animals survived and there was no swelling, rupture, or dehiscence of the wounds throughout the observation period. The wounds were observed to heal two weeks after surgery, and a small amount of newly sprouted hair was seen in the abdominal area of the rabbits. These results suggest that $\mathrm{Mg}$ clips can safely close the uterine tubes of rabbits for at least two weeks.

\subsection{In Vivo X-ray Images}

All rabbits survived the predefined implantation duration. Figure 8 shows X-ray images of the rabbits with $\mathrm{Mg}$ clips at two and three weeks after the surgery. Figure $8 \mathrm{a}-\mathrm{c}$ displays the position and condition of the clips in the rabbits two weeks after surgery. The images show that the clips could maintain their original shape during this period. Figure $8 \mathrm{~d}-\mathrm{f}$ displays the position and condition of the clips in the rabbits three weeks after surgery. The images show that the clips could maintain their grip after occlusion of the uterine tube. They also show that no significant displacement or migration occurred during this period. 


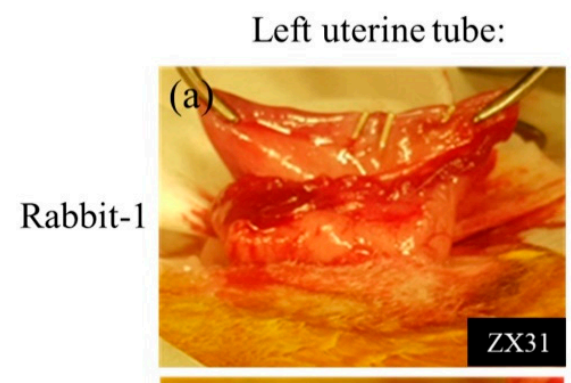

Right uterine tube:
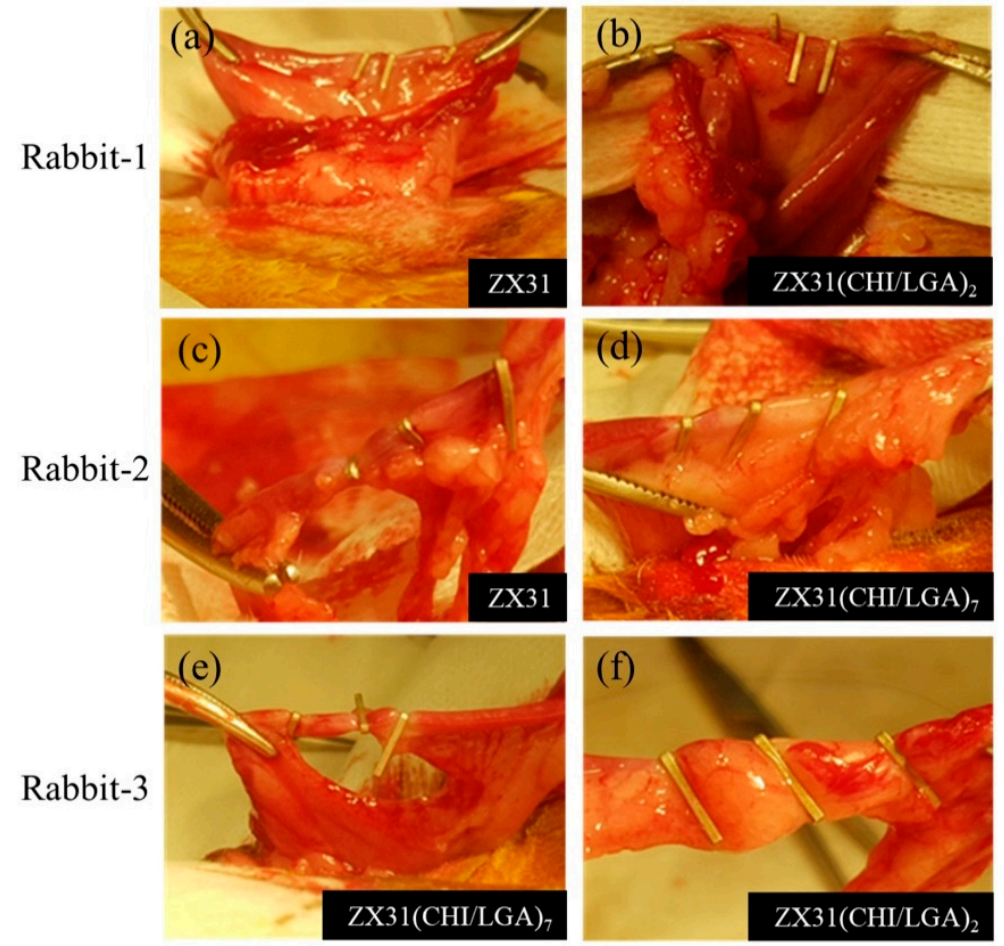

Figure 7. Placement of $\mathrm{Mg}$ clips on the uterine tubes of rabbits: $(\mathbf{a}, \mathbf{b})$ rabbit-1, for which three ZX31 and three ZX31(CHI/LGA) ${ }_{2}$ Mg clips were used; (c,d) rabbit-2, for which three ZX31 and three ZX31(CHI/LGA $)_{7} \mathrm{Mg}$ clips were used; (e,f) rabbit-3, for which three ZX31(CHI/LGA) 7 and three ZX31(CHI/LGA $)_{2} \mathrm{Mg}$ clips were used.

\section{Rabbit-1}
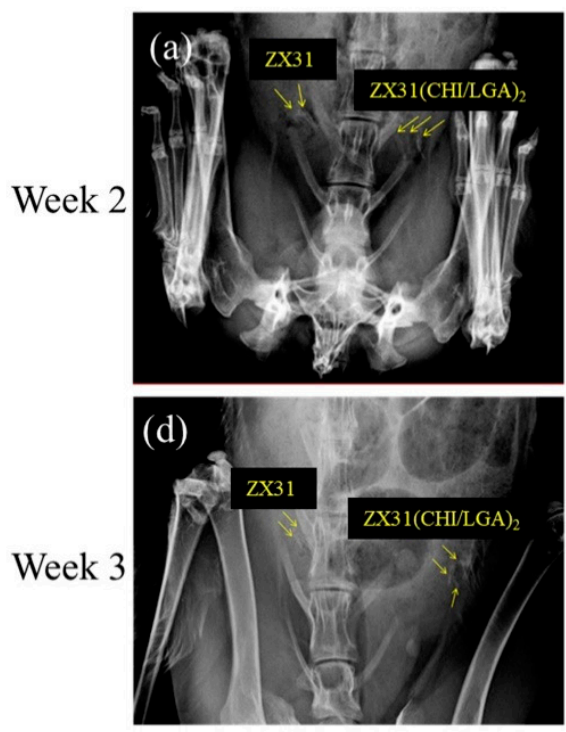

Rabbit-2
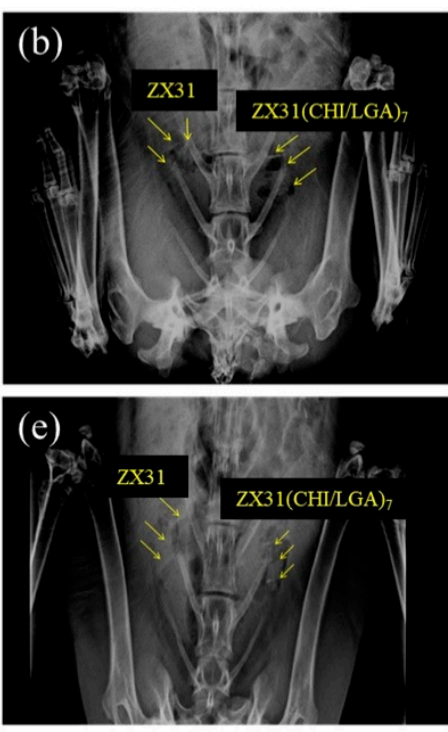

Rabbit-3
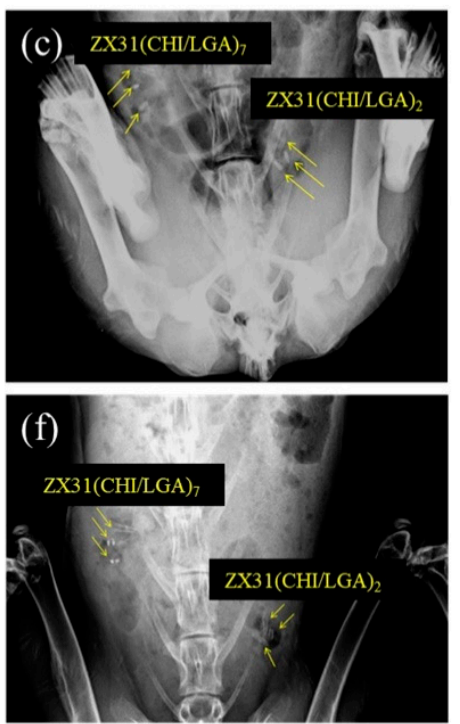

Figure 8. X-ray images of rabbits with Mg clips taken two and three weeks after surgery: (a) ZX31 and $\mathrm{ZX} 31(\mathrm{CHI} / \mathrm{LGA})_{2} \mathrm{Mg}$ clips on the left and right uterine tubes of rabbit-1, respectively (second week); (b) ZX31 and ZX31(CHI/LGA) 7 Mg clips on the left and right uterine tubes of rabbit-2, respectively (second week); (c) ZX31(CHI/LGA) 7 and ZX31(CHI/LGA) 2 Mg clips on the left and right uterine tubes of rabbit-3, respectively (second week); (d-f) the clips in the third week. 


\subsection{Autopsy and Histological Analysis}

The rabbits were euthanized under painless conditions. At the time of autopsy, no clinical abnormalities were found in the peritoneal cavity for either the coated or uncoated $\mathrm{Mg}$ clips. There was no excess production of hydrogen gas, which is the main concern preventing the practical use of $\mathrm{Mg}-\mathrm{Zn}$-Ca instruments. The excellent biocompatibility and occlusion ability of the clips were confirmed in this first stage of our study. In the next stage, it will be necessary to monitor the inosculation of actual blood vessels of animals under different conditions and diseases.

Figure 9 shows the remainder of the $\mathrm{Mg}$ clips of each group five weeks after the surgery. It can be seen that all the groups of $\mathrm{Mg}$ clips lost their original shape and function. Additionally, the weight loss values of the $\mathrm{Mg}$ clips are compared in Figure 10. The average weight loss of ZX31(CHI/LGA) clips was $4.51 \pm 0.73 \mathrm{mg}$, which was significantly lower than for both ZX31 and ZX31(CHI/LGA) ${ }_{2}$ at $5.88 \pm 0.69 \mathrm{mg}$ and $5.76 \pm 0.61 \mathrm{mg}$, respectively. This indicated that ZX31(CHI/LGA) 7 could decrease the degradation rate of the $\mathrm{Mg}-\mathrm{Zn}-\mathrm{Ca}$ alloy in vitro, but not enough to retain function.
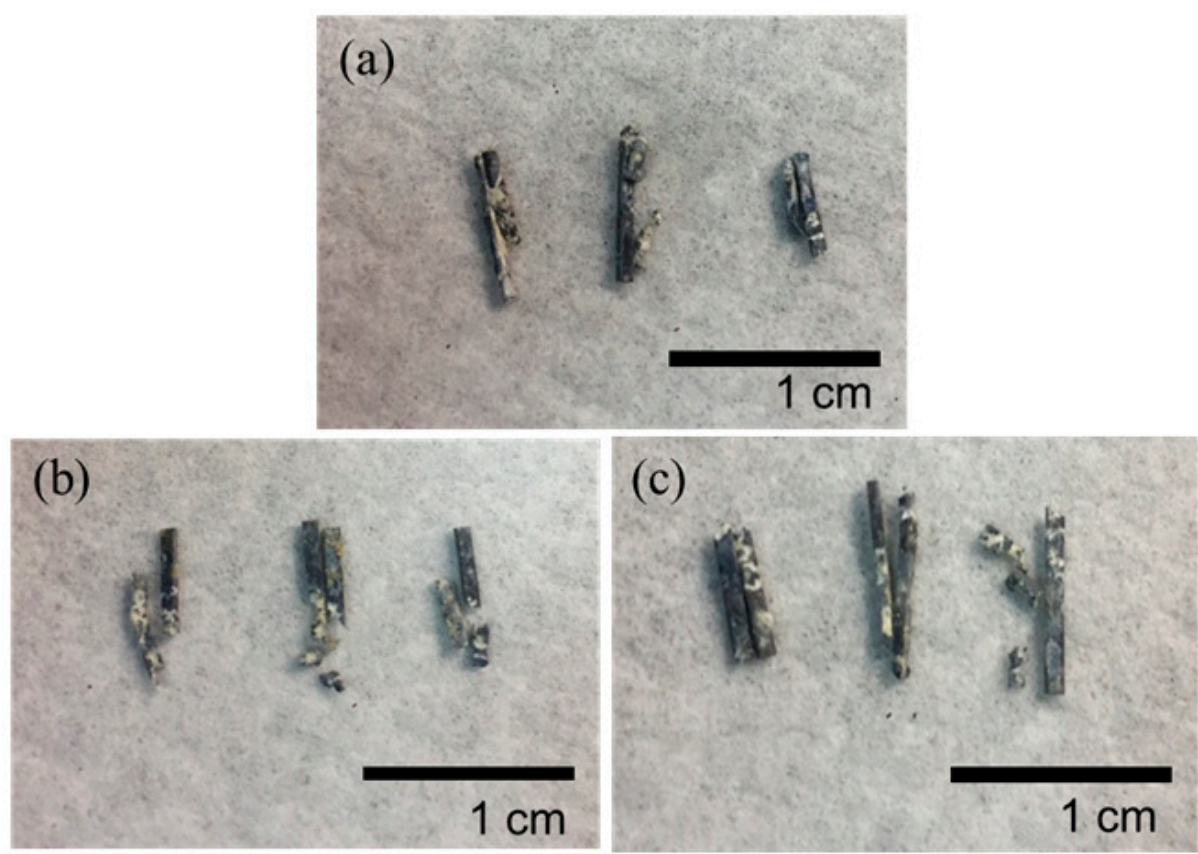

Figure 9. Mg clips taken from the rabbits five weeks after surgery: (a) ZX31 clips; (b) ZX31(CHI/LGA) clips; (c) ZX31(CHI/LGA) 7 clips.

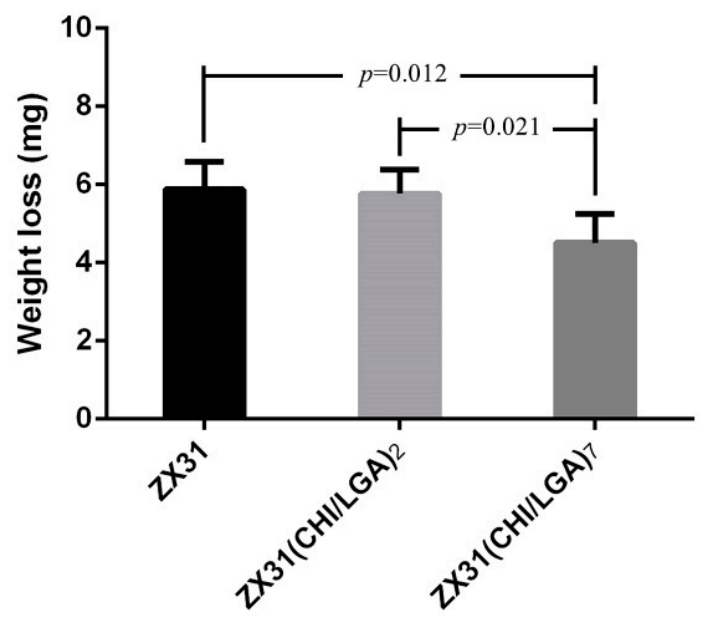

Figure 10. Weight loss of the Mg clips from each group five weeks after surgery ( $p$ values obtained via Scheffe's method). 
In this study, $\mathrm{CHI}$ coating was used to control the rate of degradation of the $\mathrm{Mg}$ clips. The in vivo experiments on animals produced better results when compared with the in vitro experiments. We speculate that this is because the $\mathrm{CHI}$ coating was present on the surface of the clips. Therefore, as the $\mathrm{CHI}$ surface layer was not absorbed because of its low degradation rate, it provided a protective effect for the $\mathrm{Mg}$ clips. It is noted that $\mathrm{ZX} 31(\mathrm{CHI} / \mathrm{LGA})_{2}$ did not present any in vitro corrosion resistance when compared with ZX31. The coating was breached because the Mg clip has many edges and corners, and the two layers of the CHI coating were, in theory, unable to effectively cover the corners and edges. Therefore, the corrosive solution seeped through the corners and edges and came into contact with the magnesium substrate beneath the coating. Consequently, the seven-layer $\mathrm{CHI}$ coating was able to provide the anticipated level of protection. However, the mechanical properties of the $\mathrm{Mg}$ clips also have to be considered. Hence, it is necessary to clarify whether micro-cracks appeared in the Mg clips after the clipping procedure, which could have accelerated their corrosion, ultimately resulting in their fracture.

The main reason why the rate of degradation of the $\mathrm{Mg}$ clips must be controlled is to minimize the effects of the release of large amounts of hydrogen [37]. Negative effects due to the release of hydrogen were not observed during our experiments. This may be because the $\mathrm{Mg}$ clips were small and the hydrogen released by the clips was simply consumed by the animals' metabolic reactions. Therefore, applications involving a large number of $\mathrm{Mg}$ clips must still be investigated in the future. A closed incisional wound takes a week to heal; thus, the clip device must be able to keep the wound closed during this time [2]. In Figure 8d-f, it is clearly shown that the various Mg clips were able to maintain closure of the wound for three weeks.

Figure 11 shows hematoxylin-and-eosin-stained, paraffin-embedded liver and kidney sections of the rabbits that were implanted with the coated $\mathrm{Mg}$ clips. No differences were observed between these rabbits and normal rabbits without $\mathrm{Mg}$ clip implantation in their liver and kidney sections. These results indicate that the $\mathrm{Mg}$ alloy with $\mathrm{CHI}$ coating showed good biocompatibility with animal organs (kidney, liver) [38]. The animals in the experiment exhibited good tolerance towards the products released due to the degradation of ZX31 and ZX31(CHI/LGA) alloy implants. Therefore, it is evident that the implanted clips are biocompatible in the early stage.

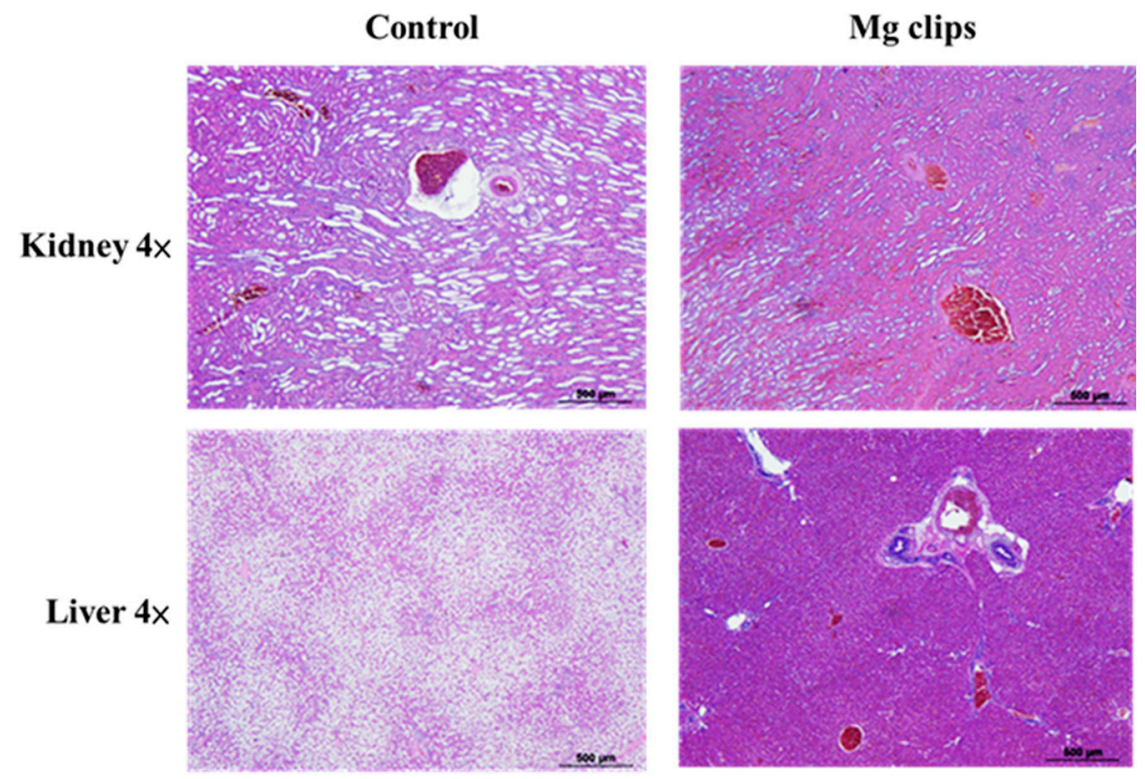

Figure 11. Histological photographs of hematoxylin-and-eosin-stained sections of tissues.

\section{Conclusions}

To achieve controllable degradation, protective and biocompatible composite layers of $\mathrm{CHI}$ and LGA were successfully electrodeposited onto an Mg-Zn-Ca surface. ZX31(CHI/LGA) 7 clips exhibited 
the lowest degradation rate in both in vitro and in vivo experiments. These coating layers acted as an effective barrier for the $\mathrm{Mg}$ substrate against corrosive environments. The specimen with the higher number of $\mathrm{CHI}$ coating layers exhibited a lower proportion of $\mathrm{Ca}$ and $\mathrm{P}$ on its surface, which means that it had a lower degradation rate. In vivo evaluation of the occlusion properties of the fabricated $\mathrm{Mg}-\mathrm{Zn}$-Ca clips showed that they could completely close the uterine tubes of rabbits. However, this did not result in significant differences between ZX31 and the coated ZX31(CHI/LGA) or ZX31(CHI/LGA $)_{7}$ specimens with regard to the function of the clips themselves. Both the uncoated and coated Mg clips exhibited excellent biocompatibility. Therefore, it can be concluded that the newly developed Mg-Zn-Ca clips are strong candidates for biodegradable soft-tissue fixation devices, such as hemostasis clips.

Author Contributions: Conceptualization, Y.-H.C. and C.C.T.; methodology, Y.-H.C. and C.-Y.C.; software, C.-H.C. and S.-Y.L.; validation, Y.-H.C., C.C.T., and J.-K.D.; formal analysis, C.-Y.C.; investigation, Y.-H.C. and J.-K.D.; resources, C.-H.C. and S.-Y.L.; data curation, C.-H.C. and S.-Y.L.; writing-original draft preparation, Y.-H.C. and J.-K.D.; writing-review and editing, J.-K.D.; visualization, C.C.T.; supervision, C.-Y.C.; project administration, J.-K.D. and C.C.T.; funding acquisition, J.-K.D. All authors have read and agreed to the published version of the manuscript.

Funding: This research received no external funding.

Acknowledgments: This work was supported by the Ministry of Science and Technology (No. MOST107-2314B-037-046-; No. MOST106-2314-B-037-013-; No. EX-03-04-07-109) and Kaohsiung Medical University Chung-Ho Memorial Hospital (No. KMUH105-5R63; No. KMUH106-6R73; No. KMUH107-7R76). We thank the Metal Industries Research and Development Centre for helping with the production of the hemostatic clips.

Conflicts of Interest: The authors declare no conflict of interest.

\section{References}

1. Yoshida, T.; Fukumoto, T.; Urade, T.; Kido, M.; Toyama, H.; Asari, S.; Ajiki, T.; Ikeo, N.; Mukai, T.; Ku, Y. Development of a new biodegradable operative clip made of a magnesium alloy: Evaluation of its safety and tolerability for canine cholecystectomy. Surgery 2017, 161, 1553-1560. [CrossRef]

2. Ikeo, N.; Nakamura, R.; Naka, K.; Hashimoto, T.; Yoshida, T.; Urade, T.; Fukushima, K.; Yabuuchi, H.; Fukumoto, T.; Ku, Y.; et al. Fabrication of a magnesium alloy with excellent ductility for biodegradable clips. Acta Biomater. 2016, 29, 468-476. [CrossRef]

3. Ito, K.; Seguchi, T.; Nakamura, T.; Chiba, A.; Hasegawa, T.; Nagm, A.; Horiuchi, T.; Hongo, K. Evaluation of Metallic Artifacts Caused by Nonpenetrating Titanium Clips in Postoperative Neuroimaging. World Neurosurg. 2016, 96, 16-22. [CrossRef] [PubMed]

4. Shaker, T.; Hackett, T. A case of choledocholithiasis secondary to post cholecystectomy clip migration. Eur. J. Mol. Clin. Med. 2017, 2, 26. [CrossRef]

5. Guilbaud, T.; Scemama, U.; Lestelle, V.; Orsoni, P.C.; Birnbaum, D.J. Unfortunate adverse event resulting from clip migration after cholecystectomy. Surgery 2019, 165, 851-852. [CrossRef]

6. Antunes, A.G.; Peixe, B.; Guerreiro, H. Pancreatitis and cholangitis following intraductal migration of a metal clip 5 years after laparoscopic cholecystectomy. Gastroenterol. Y Hepatol. 2017, 40, 615-617. [CrossRef] [PubMed]

7. Staiger, M.P.; Pietak, A.M.; Huadmai, J.; Dias, G. Magnesium and its alloys as orthopedic biomaterials: A review. Biomaterials 2006, 27, 1728-1734. [CrossRef] [PubMed]

8. Jafari, S.; Singh Raman, R.K. In-vitro biodegradation and corrosion-assisted cracking of a coated magnesium alloy in modified-simulated body fluid. Mater. Sci. Eng. C 2017, 78, 278-287. [CrossRef]

9. Witte, F.; Hort, N.; Vogt, C.; Cohen, S.; Kainer, K.U.; Willumeit, R.; Feyerabend, F. Degradable biomaterials based on magnesium corrosion. Curr. Opin. Solid State Mater. Sci. 2008, 12, 63-72. [CrossRef]

10. Peron, M.; Skaret, P.C.; Fabrizi, A.; Varone, A.; Montanari, R.; Roven, H.J.; Ferro, P.; Berto, F.; Torgersen, J. The effect of Equal Channel Angular Pressing on the stress corrosion cracking susceptibility of AZ31 alloy in simulated body fluid. J. Mech. Behav. Biomed. Mater. 2020. [CrossRef]

11. Gu, X.; Zheng, Y.; Cheng, Y.; Zhong, S.; Xi, T. In vitro corrosion and biocompatibility of binary magnesium alloys. Biomaterials 2009, 30, 484-498. [CrossRef] 
12. Cui, L.-Y.; Xu, J.; Lu, N.; Zeng, R.-C.; Zou, Y.-h.; Li, S.-Q.; Zhang, F. In vitro corrosion resistance and antibacterial properties of layer-by-layer assembled chitosan/poly-L-glutamic acid coating on AZ31 magnesium alloys. Trans. Nonferrous Met. Soc. China 2017, 27, 1081-1086. [CrossRef]

13. Xia, Y.H.; Zhang, B.P.; Lu, C.X.; Geng, L. Improving the corrosion resistance of Mg-4.0Zn-0.2Ca alloy by micro-arc oxidation. Mater. Sci. Eng. C 2013, 33, 5044-5050. [CrossRef] [PubMed]

14. Zhao, Y.-B.; Liu, H.-P.; Li, C.-Y.; Chen, Y.; Li, S.-Q.; Zeng, R.-C.; Wang, Z.-L. Corrosion resistance and adhesion strength of a spin-assisted layer-by-layer assembled coating on AZ31 magnesium alloy. Appl. Surf. Sci. 2018, 434, 787-795. [CrossRef]

15. Pozzo, L.D.; da Conceição, T.F.; Spinelli, A.; Scharnagl, N.; Pires, A.T. Chitosan coatings crosslinked with genipin for corrosion protection of AZ31 magnesium alloy sheets. Carbohydr. Polym. 2018, 181, 71-77. [CrossRef] [PubMed]

16. Wan, P.; Tan, L.; Yang, K. Surface Modification on Biodegradable Magnesium Alloys as Orthopedic Implant Materials to Improve the Bio-adaptability: A Review. J. Mater. Sci. Technol. 2016, 32, 827-834. [CrossRef]

17. Peron, M.; Bin Afif, A.; Dadlani, A.; Berto, F.; Torgersen, J. Comparing physiologically relevant corrosion performances of $\mathrm{Mg}$ AZ31 alloy protected by $\mathrm{ALD}$ and sputter coated $\mathrm{TiO}_{2}$. Surf. Coat. Technol. 2020. [CrossRef]

18. Li, H.; Peng, L. Antimicrobial and antioxidant surface modification of cellulose fibers using layer-by-layer deposition of chitosan and lignosulfonates. Carbohydr. Polym. 2015, 124, 35-42. [CrossRef]

19. Ruvinov, E.; Cohen, S. Alginate biomaterial for the treatment of myocardial infarction: Progress, translational strategies, and clinical outlook: From ocean algae to patient bedside. Adv. Drug Deliv. Rev. 2016, 96, 54-76. [CrossRef]

20. Daskalova, A.; Nathala, C.S.R.; Bliznakova, I.; Stoyanova, E.; Zhelyazkova, A.; Ganz, T.; Lueftenegger, S.; Husinsky, W. Controlling the porosity of collagen, gelatin and elastin biomaterials by ultrashort laser pulses. Appl. Surf. Sci. 2014, 292, 367-377. [CrossRef]

21. Ahmed, E.M. Hydrogel: Preparation, characterization, and applications: A review. J. Adv. Res. 2015, 6, 105-121. [CrossRef] [PubMed]

22. Hamedi, H.; Moradi, S.; Hudson, S.M.; Tonelli, A.E. Chitosan based hydrogels and their applications for drug delivery in wound dressings: A review. Carbohydr. Polym. 2018, 199, 445-460. [CrossRef] [PubMed]

23. Chen, Y.; Yan, X.; Zhao, J.; Feng, H.; Li, P.; Tong, Z.; Yang, Z.; Li, S.; Yang, J.; Jin, S. Preparation of the chitosan/poly(glutamic acid)/alginate polyelectrolyte complexing hydrogel and study on its drug releasing property. Carbohydr. Polym. 2018, 191, 8-16. [CrossRef]

24. Tan, H.; Chu, C.R.; Payne, K.A.; Marra, K.G. Injectable in situ forming biodegradable chitosan-hyaluronic acid based hydrogels for cartilage tissue engineering. Biomaterials 2009, 30, 2499-2506. [CrossRef] [PubMed]

25. Ahsan, S.M.; Thomas, M.; Reddy, K.K.; Sooraparaju, S.G.; Asthana, A.; Bhatnagar, I. Chitosan as biomaterial in drug delivery and tissue engineering. Int. J. Biol. Macromol. 2018, 110, 97-109. [CrossRef]

26. Akilbekova, D.; Shaimerdenova, M.; Adilov, S.; Berillo, D. Biocompatible scaffolds based on natural polymers for regenerative medicine. Int. J. Biol. Macromol. 2018, 114, 324-333. [CrossRef]

27. Zhao, N.; Yang, C.; Wang, Y.; Zhao, B.; Bian, F.; Li, X.; Wang, J. Probing the surface microstructure of layer-by-layer self-assembly chitosan/poly(l-glutamic acid) multilayers: A grazing-incidence small-angle X-ray scattering study. Mater. Sci. Eng. C 2016, 58, 352-358. [CrossRef]

28. Park, K.; Jeong, H.; Tanum, J.; Yoo, J.-C.; Hong, J. Poly-1-lysine/poly-l-glutamic acid-based layer-by-layer self-assembled multilayer film for nitric oxide gas delivery. J. Ind. Eng. Chem. 2018, 69, 263-268. [CrossRef]

29. Lih, E.; Lee, J.S.; Park, K.M.; Park, K.D. Rapidly curable chitosan-PEG hydrogels as tissue adhesives for hemostasis and wound healing. Acta Biomater. 2012, 8, 3261-3269. [CrossRef]

30. Jiang, P.L.; Hou, R.Q.; Chen, C.D.; Sun, L.; Dong, S.G.; Pan, J.S.; Lin, C.J. Controllable degradation of medical magnesium by electrodeposited composite films of mussel adhesive protein (Mefp-1) and chitosan. J Colloid Interface Sci. 2016, 478, 246-255. [CrossRef]

31. Liangjian, C.; Jun, Z.; Kun, Y.; Chang, C.; Yilong, D.; Xueyan, Q.; Zhiming, Y. Improving of in vitro Biodegradation Resistance in a Chitosan Coated Magnesium Bio-composite. Rare Met. Mater. Eng. 2015, 44, 1862-1865. [CrossRef]

32. Chen, S.; Tu, J.; Hu, Q.; Xiong, X.; Wu, J.; Zou, J.; Zeng, X. Corrosion resistance and in vitro bioactivity of Si-containing coating prepared on a biodegradable Mg-Zn-Ca bulk metallic glass by micro-arc oxidation. J. Non-Cryst. Solids 2017, 456, 125-131. [CrossRef] 
33. Wong, P.-C.; Tsai, P.-H.; Li, T.-H.; Cheng, C.-K.; Jang, J.S.C.; Huang, J.C. Degradation behavior and mechanical strength of $\mathrm{Mg}-\mathrm{Zn}-\mathrm{Ca}$ bulk metallic glass composites with Ti particles as biodegradable materials. J. Alloy Compd. 2017, 699, 914-920. [CrossRef]

34. Fang, J.; Zhang, Y.; Yan, S.; Liu, Z.; He, S.; Cui, L.; Yin, J. Poly(l-glutamic acid)/chitosan polyelectrolyte complex porous microspheres as cell microcarriers for cartilage regeneration. Acta Biomater. 2014, 10, $276-288$. [CrossRef]

35. Bai, K.; Zhang, Y.; Fu, Z.; Zhang, C.; Cui, X.; Meng, E.; Guan, S.; Hu, J. Fabrication of chitosan/magnesium phosphate composite coating and the in vitro degradation properties of coated magnesium alloy. Mater. Lett. 2012, 73, 59-61. [CrossRef]

36. Pan, Y.K.; Chen, C.Z.; Wang, D.G.; Zhao, T.G. Improvement of corrosion and biological properties of microarc oxidized coatings on $\mathrm{Mg}-\mathrm{Zn}-\mathrm{Zr}$ alloy by optimizing negative power density parameters. Colloids Surf. B Biointerfaces 2014, 113, 421-428. [CrossRef]

37. Thormann, U.; Alt, V.; Heimann, L.; Gasquere, C.; Heiss, C.; Szalay, G.; Franke, J.; Schnettler, R.; Lips, K.S. The biocompatibility of degradable magnesium interference screws: An experimental study with sheep. Biomed. Res. Int. 2015, 2015, 1-15. [CrossRef]

38. Hou, L.; Li, Z.; Pan, Y.; Du, L.; Li, X.; Zheng, Y.; Li, L. In vitro and in vivo studies on biodegradable magnesium alloy. Prog. Nat. Sci. Mater. Int. 2014, 24, 466-471. [CrossRef]

(C) 2020 by the authors. Licensee MDPI, Basel, Switzerland. This article is an open access article distributed under the terms and conditions of the Creative Commons Attribution (CC BY) license (http://creativecommons.org/licenses/by/4.0/). 